increased by $11 \%$ and $17 \%$, respectively, after four months. Job facilitating conditions and job satisfaction also demonstrated statistically significant improvement at four months $(\mathrm{p}=0.01$ and $\mathrm{p}=0.04$, respectively).

Concluion An intranet website for knowledge sharing can be an easily-deployed and inexpensive way to support improvement in job facilitation, self-efficacy, and job satisfaction in NIR. Such a website is likely to be beneficial in other dynamic procedural environments without dedicated staff. The study was limited by lack of controls for other work environment changes, such as staff turn-over, anonymous data collection preventing cross-linking of individual respondents across time, and utilization of self-reported adoption rates.

Disclosures Z. Voronovich: None. A. Montes: None. D. Sorte: None.

\section{E-079 TIME OF DAY AND ENDOVASCULAR TREATMENT DECISION IN ACUTE STROKE - INSIGHTS FROM AN INTERNATIONAL MULTIDISCIPLINARY SURVEY}

${ }^{1} \mathrm{~N}$ Kashani ${ }^{*},{ }^{2} \mathrm{~J}$ Ospel, ${ }^{1} \mathrm{~A}$ Wilson, ${ }^{3} \mathrm{~W}$ Kunz, ${ }^{4} \mathrm{P}$ Sylaia, ${ }^{5} \mathrm{~B}$ Baxter, ${ }^{6} \mathrm{~B}$ Campbell, ${ }^{7} \mathrm{U}$ Fischer, ${ }^{8} \mathrm{~A}$ Rabinstein, ${ }^{9} \mathrm{~S}$ Yoshimura, ${ }^{10} \mathrm{~J}$ Heo, ${ }^{11} \mathrm{~B}$ Kim, ${ }^{12} \mathrm{M}$ Cherian, ${ }^{13} \mathrm{~F}$ Turjman, ${ }^{1} \mathrm{M}$ Foss, ${ }^{1} \mathrm{~B}$ Menon, ${ }^{14} \mathrm{G}$ Saposnik, ${ }^{1} \mathrm{M}$ Goyal. ${ }^{1}$ Radiology, University of Calgary, Calgary, $A B$, Canada; ${ }^{2}$ Radiology, University Hospital Basel, Basel, Switzerland; ${ }^{3}$ Radiology, University Hospital Munich, Munich, GERMANY; ${ }^{4}$ Radiology, University of Calgary, Thiruvananthapuram, India; ${ }^{5}$ Radiology, Erlanger Hospital Chattanooga, Chattanooga, TN; ${ }^{6}$ Radiology, Royal Melbourne Hospital, University of Melbourne, Melbourne, Australia; ${ }^{7}$ Radiology, University Hospital Bern (Inselspital), Bern, Switzerland; ${ }^{8}$ Radiology, Mayo Clinic Rochester, Calgary, MN; ${ }^{9}$ Radiology, University of Calgary, Hyogo, Japan; ${ }^{10}$ Neurology, Yonsei University, Seoul, Korea, republic of; ${ }^{11}$ Radiology, St. Mary's Hospital Seoul, Calgary, Korea, republic of; ${ }^{12}$ Radiology, University of Calgary, Tamil Nadu, India; ${ }^{13}$ Radiology, Centre Hospitalier Universitaire de Lyon, Lyon, France; ${ }^{14}$ Radiology, University of Toronto, Calgary, ON, Canada

\subsection{6/neurintsurg-2019-SNIS.154}

Background The decision to proceed with endovascular thrombectomy should ideally be made independent of inconvenience factors such as daytime, especially in cases supported by level IA evidence. The treatment decision may be influenced by the time of day when facing level 2B evidence cases. We assessed the influence of patient presentation time on EVT decision- making under current local resources and assumed ideal conditions.

Methods In an international cross-sectional survey among 607 stroke physicians, participants were asked to give their treatment decisions to 10 out of 22 randomly assigned case scenarios, 8 of them were level $1 \mathrm{~A}$ evidence cases (all daytime scenarios), 11 were scenarios where level $2 \mathrm{~B}$ evidence supports EVT, among them 7 daytime scenarios (8:00 am5:00 pm) and 4 overnight cases (10 pm:00 - 7:00 am). The remaining 3 ( 2 daytime and 1 nighttime) cases were not covered by current treatment guidelines. Participants stated A) their treatment approach assuming there were no practice constraints, and B) the treatment they would pursue under their current local resources. Endovascular treatment decision in level $2 \mathrm{~B}$ scenarios was analyzed with regard to presentation time.

Results 3034 responses for 12 case scenarios from 607 physicians from 38 countries with level $2 \mathrm{~B}$ evidence and 2208 for level 1A evidence for EVT were received. In the subgroup of level 2B evidence scenarios, participants selected EVT more often in the overnight cases (in $74.2 \%$ under assumed ideal conditions and in $70.7 \%$ under current local resources) than in the daytime scenarios (in $67.2 \%$ and $63.8 \%$ respectively). Overnight presentation did not increase the risk for a treatment decision against EVT. Current nighttime EVT decision rates for level $2 \mathrm{~B}$ scenarios in 7 countries were $>10 \%$ lower than ideal nighttime EVT decision rates.

Conclusion Overall, overnight presentation did not increase the risk of a treatment decision against EVT when facing level 2B evidence. Hence, presentation time is unlikely to influence endovascular treatment decision-making in this subset of stroke patients. Current nighttime EVT decision rates in 7 countries were substantially lower than ideal nighttime EVT rates, potentially indicating limited access to endovascular treatment beyond working hours.

Disclosures N. Kashani: None. J. Ospel: None. A. Wilson: None. W. Kunz: None. P. Sylaia: None. B. Baxter: None. B. Campbell: None. U. Fischer: None. A. Rabinstein: None. S. Yoshimura: None. J. Heo: None. B. Kim: None. M. Cherian: None. F. Turjman: None. M. Foss: None. B. Menon:None. G. Saposnik: None. M. Goyal: None.
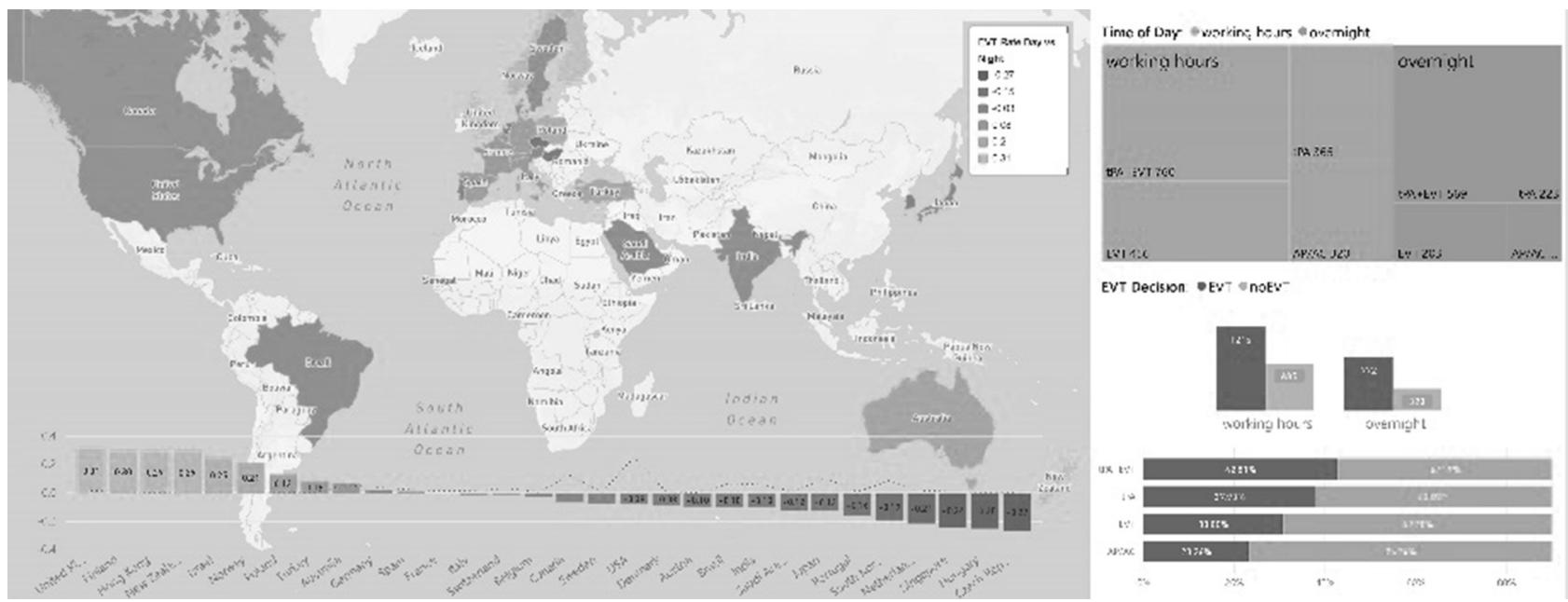

Abstract E-079 Figure 1 